\title{
BMJ Open Exercise improves sleep quality in older adults: a protocol for a systematic review and meta-analysis
}

\author{
Wei Zhang (1) , ,2,3,4 Yi Liu, ${ }^{1,2,3,4}$ Jing Yu, ${ }^{2,3,4}$ Dongze Li, ${ }^{1}{ }^{\text {Yu Jia, }}{ }^{1}$ Qin Zhang, ${ }^{1,2,3,4}$ \\ Yongli Gao, ${ }^{1,2,3,4}$ Zhi Wan, ${ }^{1}$ Wei Wei ${ }^{1}$
}

To cite: Zhang W, Liu Y, Yu J, et al. Exercise improves sleep quality in older adults: a protocol for a systematic review and meta-analysis. BMJ Open 2022;12:e047555. doi:10.1136/ bmjopen-2020-047555

- Prepublication history and additional supplemental material for this paper are available online. To view these files, please visit the journal online (http://dx.doi.org/10.1136/ bmjopen-2020-047555).

Received 02 December 2020 Accepted 07 January 2022

Check for updates

(C) Author(s) (or their employer(s)) 2022. Re-use permitted under CC BY-NC. No commercial re-use. See rights and permissions. Published by BMJ.

${ }^{1}$ Department of Emergency Medicine, Disaster Medical Center, West China Hospital, Sichuan University, Chengdu, People's Republic of China

${ }^{2}$ School of Nursing, West China Hospital, Sichuan University, Chengdu, People's Republic of China

${ }^{3}$ Institute of Disaster Medicine, Sichuan University, Chengdu, People's Republic of China ${ }^{4}$ Nursing Key Laboratory of Sichuan Province, Chengdu, People's Republic of China

Correspondence to Professor Wei Wei; ww8075@126.com and Professor Zhi Wan; 303680215@qq.com

\section{ABSTRACT}

Introduction Sleep disturbance is a common problem among the elderly and is associated with physical and mental health. Exercise has been reported as an alternative therapeutic strategy for people with sleep disturbances. However, this topic has not been systematically reviewed for older adults. This study was conducted to provide a protocol to systematically evaluate the effects of exercise on sleep quality in the elderly. Methods and analysis An electronic search of the PubMed, Embase and Cochrane Library databases will be performed with no language restrictions, and data extraction will be performed by two independent reviewers. The reviewers will discuss and resolve any differences, and a third reviewer will be consulted in cases of uncertainty. Randomised controlled trials will be selected. The primary outcome will be an objective measurement of sleep quality (eg, polysomnography). The secondary outcomes will be self-reported sleep quality (using the Pittsburgh Sleep Quality Index scale), and adverse events (such as falls and fractures). RevMan V.5.3.5 and Stata V.16.0 software will be used for metaanalysis. If the heterogeneity tests show slight or no statistical heterogeneity, the fixed effects model will be used; in other cases, the random effect model will be used for data synthesis.

Ethics and dissemination The protocol does not require ethical approval. The findings will be disseminated in peerreviewed publications and journals.

PROSPERO registration number CRD42021287980.

\section{INTRODUCTION}

Sleep plays an important part in the maintenance and promotion of health, accounting for nearly one-third of human lives. Sleep disorders can manifest as problems of either insufficient sleep, abnormal movements during sleep or excessive amount of perceived sleep. ${ }^{1}$ It can be caused by variety of factors, often associated with physical diseases. The International Classification of Sleep disturbancess (ICSD-3) ${ }^{2}$ identifying seven major categories that include insomnia disorders, sleep-related breathing disorders, central disorders of hypersomnolence, circadian rhythm sleep-wake disorders. The

\section{Strengths and limitations of this study}

- This study will provide an optimal exercise model suitable for the elderly with sleep disturbances by identifying the intervention effectiveness of exercise for sleep disturbances in the old.

- This protocol follows the Preferred Reporting Items for Systematic Review and Meta-Analysis Protocols guidelines.

- Two independent reviewers will identify studies for inclusion and perform data extraction, and the third author will address any disagreements.

- There will be have language restriction in the selection of the studies.

proportion of adults in the USA who have less than 6 hours of sleep a night has increased by $31 \%$ since $1985 .{ }^{3}$ In Canada, more than $40 \%$ of people have sleep disturbances. ${ }^{4}$ Qualitative and quantitative changes in sleep patterns occur with increasing age, and it is estimated that more than $50 \%$ of older adults have sleep disturbances. ${ }^{56}$ Studies have shown that sleep disturbance is a potential risk factor for major health problems. ${ }^{78}$ Sleep disturbances may be related to several mental and cognitive disorders including persistent delusions, ${ }^{9}$ depression, anxiety and dementia ${ }^{10}$ and often coexist with major medical conditions, such as hypertension, ${ }^{11}{ }^{12}$ diabetes ${ }^{13}$ and cancer, ${ }^{14}$ which may increase pain and even increase the risk of mortality. ${ }^{15} 16$ Therefore, sleep disturbance is an important therapeutic target for healthcare.

The American Psychiatric Association recommends three treatments for insomnia: pharmacological therapy, psychotherapy and complementary and alternative therapy. ${ }^{17}$ Among these, pharmacological therapy is the most common treatment. ${ }^{18}$ The use of sleep-inducing drugs can lead to negative effects such as drowsiness, gait disorders and cognitive impairment. ${ }^{19}$ Older adults with an underlying disease may take a large 
number of other drugs in addition to medications for insomnia. Existing evidence suggests that the use of these drugs may increase the risk of falls and fractures in older adults, ${ }^{202}$ and long-term use of the drugs can cause withdrawal syndromes associated with its administration and dependence. ${ }^{22}$ It is, therefore, necessary to discover an effective non-drug treatment for insomnia.

Age-related declines in physical activity and sleep are both important health indicators for successful 'healthy ageing'. Exercise intervention began to receive attention. Previous studies showed ${ }^{23}$ that regular exercise offers many health benefits, including reduced risk of chronic disease, all-cause mortality, and premature death. Because comparing with traditional drug therapy, exercise is a low-cost alternative therapeutic strategy with few adverse events. Present studies have shown an association between exercise and sleep, mediated by a variety of psychological and physiological pathways, and that moderate exercise can improve sleep quality. ${ }^{24}$ Due to the different forms of exercise, durations, frequencies and cycles of the exercise interventions, the effects differ. Previous meta-analyses have drawn contradictory conclusions about the effects of exercise on sleep. ${ }^{25-29}$ And these only studied the effects of a single or a few types of exercise on sleep. The question of which type of exercise is most suitable for older adults was not investigated. It is also not clear how the duration, frequency and intensity of exercise affect sleep quality in the elderly. Moreover, these studies did not explore differences in the effects of exercise on different types of sleep disturbances. The aetiology and clinical manifestations are different for different sleep disturbances, so it is very necessary to develop personalised exercise pattern for the elderly with sleep disturbances. Furthermore, additional studies have appeared since the publication of these meta-analyses and this new evidence from other randomised trials should be considered. Our aim, therefore, is to synthesis the available evidence and conduct a high-quality meta-analysis to compare the effects of different exercise patterns on sleep quality in the elderly.

We have asked four primary questions in this study:

1. Which type of exercise intervention is most effective for older adults with sleep disturbances?

2. How does the exercise duration, frequency and intensity mediate/moderate the exercise-sleep relationship?

3. Does exercise affect the elderly in other ways (mental health, physical health, cognitive function)?

4. For different sleep disturbances, which type of exercise intervention is most effective?

\section{METHODS AND ANALYSIS}

This study follows the reporting guidelines of the Preferred Reporting Items for Systematic Review and Meta-Analysis Protocols 2015 (PRISMA-P). The research method uses the guidelines described in the Cochrane Handbook for Systematic Reviews of Diagnostic Test Accuracy.

\section{Eligibility criteria}

Studies that meet all of the following criteria will be included in the meta-analysis.

\section{Types of studies}

Randomised controlled trials (RCTs) which compare different exercise methods on the sleep quality of the elderly (eg, Tai Chi, yoga, Qigong, Bagua, aerobic exercise, resistance exercise and a combination of aerobic and resistance exercise) will be included. The study must include at least one self-report or observable measure of sleep. Other types of research, such as observational studies and animal trials, will be excluded. A flow chart of the study selection process is shown in figure 1 .

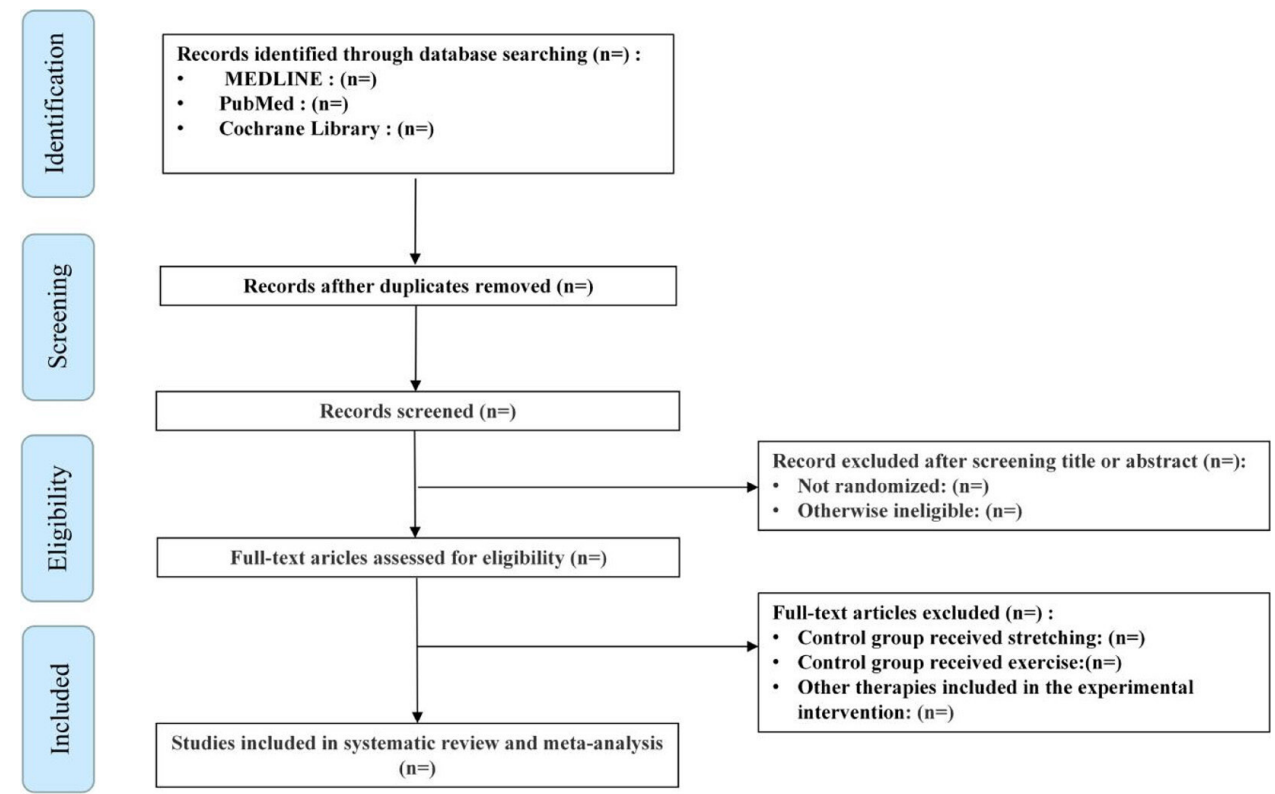

Figure 1 Flow diagram of study selection. 


\section{Types of participants}

Participants will be included irrespective of country of origin, age, race and gender. People over 60 years old diagnosed with sleep disturbances (according to the ICSD-3) will be included. Samples of clinical populations such as individuals with a diagnosis of dementia or Parkinson's disease will be excluded.

\section{Types of interventions}

We will include RCTs which interventions that meet the above criteria and include at least one exercise intervention. An intervention period of at least 1 month was chosen.

The eligible types of exercise will be the following:

- Tai Chi.

- Qigong.

- Yoga.

- Bagua.

- Aerobic exercise (walking, brisk walking, jogging, race walking, skating, long-distance swimming, cycling, ball game, etc).

- Resistance exercise (push-ups, dumbbells, barbells, etc).

- Combination of aerobic exercise and resistance exercise.

- Combination of aerobic exercise and resistance exercise (Tai Chi, Qigong, Yoga, Bagua).

Types of outcome measures

Primary outcome: objective assessment of sleep quality (eg, polysomnography, sleep onset latency, sleep duration, sleep disturbance, habitual sleep efficiency, daytime dysfunction and use of sleep medication).

Secondary outcome: subjective assessment of sleep quality (eg, the Pittsburgh Sleep Quality Index (PSQI)) and adverse events (eg, muscle sprains and pains, myocardial infarction, stroke).

\section{Information sources}

Two investigators (YL and ZW) will search studies in PubMed, Embase and the Cochrane Library using keywords for sleep disturbances (sleep disturbance/s, sleep quality, insomnia, sleep problems, sleep disorder) and for exercise (Tai Chi, Qigong, Yoga, Bagua, Aerobic exercise, Resistance exercise, Combination of aerobic exercise and resistance exercise, exercise, physical activity, physical therapy) form their inception up to December 2021 (see online supplemental file 1. Results of the literature retrieval in PubMed). The retrieval words will be subject words plus free words, which are determined after repeated prechecking, supplemented by manual retrieval. The study will limit the search results to articles published in Chinese and English. To prevent omissions, we will retrieve the references twice.

\section{Data collection and analysis}

Selection of studies

The Endnote V.X9.3.3 software will be used to manage records. A double-blind method will be used for literature screening by the two investigators (YL and ZW). First, for the preliminary screening, we will read the title and abstract of the article. After removing duplicates both manually and electronically, the overall accuracy of the search will be calculated by dividing the number of studies that meet the eligibility criteria by the total number of studies after the removal of duplicates. After the screening, YL and ZW will compare the extracted literature. Additionally, relevant articles cited in the included studies will be entered into the full-text screening. YL and ZW will independently screen the titles and abstracts of all the studies and will determine inclusion or exclusion according to the above criteria. All disagreements will be discussed and resolved by $\mathrm{YL}$ and $\mathrm{ZW}$, and a third author (JY) if necessary. The details of the study selection and identification process will be presented in a flowchart (figure 1).

\section{Data collection and management}

After the study selection, the two independent investigators $(\mathrm{YL}$ and $\mathrm{ZW})$ will collect all the essential data from each eligible study. If data information is lacking or unclear, the investigators will contact the original author by email. The contents of the data extraction include (1) basic data (first author, publication year, country, sample size, age and distribution mode of subjects); (2) participant characteristics (age, sex, race, country, basic illness, type of used drug and type of sleep disturbance); (3) experimental characteristics (type of exercise, intervention measures, experimental cycle, duration, frequency and control intervention mode); and (4) outcome indicators (objective measurement results, subjective measurement results and adverse events). The outcome indicators we will consider include both objective and subjective measurement results. Objective measurement results include polysomnography, insomnia duration, sleep disturbances, habitual sleep efficiency, sleep latency, daytime dysfunction and the use of sleeping pills. $\mathrm{PSQI}^{30}$ will be employed as the primary outcome measurement. The PSQI consists of 19 questions generating 7 components, each with a score from 0 to 3 . The seven scores are added together to generate a global PSQI score (ranging from 0 to 21). A score greater than 5 indicates clinical sleep disturbances. The adverse events refer to falls, fractures and death. Other standardised instruments or scales, such as the Self-Rating Scale of Sleep, ${ }^{31}$ Athens Insomnia Scale ${ }^{32}$ and the Insomnia Severity Index ${ }^{33}$ will be included. The adverse events refer to muscle sprains and pains, myocardial infarction and stroke. In the event of a dispute over the included data, the two investigators will discuss and resolve the problem and, if necessary, the third researcher (JY) will join the discussion.

\section{Statistical analysis}

\section{Study quality assessment}

Two investigators ( $\mathrm{QZ}$ and JY) will conduct a quality assessment of each selected study according to the quality evaluation criteria of RCTs in Cochrane Collaboration's tool. 
This includes the random sequence allocation of participants and whether this was truly hidden from the investigators, blinding methods for subjects and researchers, the blinding method for outcome evaluation, the presence of incomplete outcome data, selective reporting, and other considerations. Each study will be evaluated as 'low bias risk', 'bias uncertainty' or 'high bias risk'. Any disagreements in the abstracted data will be adjudicated by a third investigator (DL).

\section{Assessment of heterogeneity}

If the data are available, a heterogeneity analysis will be performed. The $\chi^{2}$ test (test level: $\alpha=0.1$ ) and $\mathrm{I}^{2}$ will be used to test heterogeneity. If $\mathrm{p}>0.01$, there is no heterogeneity among the studies. If $\mathrm{p}<0.01$, there is heterogeneity among the studies. $\mathrm{I}^{2}$ represents the level of heterogeneity between studies: if $\mathrm{I}^{2}$ is between $0 \%$ and $40 \%$, the heterogeneity is negligible, if $\mathrm{I}^{2}$ is between $40 \%$ and $60 \%$, there is moderate heterogeneity, if $\mathrm{I}^{2}$ is between $60 \%$ and $75 \%$, there is high heterogeneity and if $\mathrm{I}^{2}$ is between $75 \%$ and $100 \%$, this indicates very high heterogeneity.

\section{Assessment of reporting bias}

The Stata V.16 software package will be used for the assessment of reporting bias. If there are sufficient data, we will use funnel charts and the Egger test to assess the likelihood of research bias in small studies. Asymmetry in the funnel chart indicates bias with the more obvious the asymmetry, the greater the degree of bias.

\section{Outcome measures}

RevMan V.5.3.5 software (Cochrane, London, UK) will be used for the meta-analysis. We will provide descriptive statistics on the available data from experimental studies and demographic characteristics, as well as important variables (age, follow-up time, outcome-related baseline risk factors, underlying diseases). Calculating the score or score change after exercise intervention will standardise the mean difference (SMD) and 95\% CI. SMD of 0.5 means that the average value of the exercise group is more than half the $\mathrm{SD}$ of the control group. $\mathrm{SMD} \geq 0.8$ is regarded as large, SMD is $0.5-0.79$ is medium, SMD is $0.2-0.49$ is small, $\mathrm{SMD}<0.2$ is insignificant. A double tail $\alpha \leq 0.05$ will be considered statistically significant. Certain secondary outcomes, for example, adverse events, belong to dichotomous variables. If the data are available, the risk ratio or OR with $95 \%$ CIs will be calculated for these secondary outcomes.

If the results are statistically significant, the $95 \%$ prediction interval (PI) will be calculated. The PI can be used to approximate the therapeutic effect in the new study, ${ }^{34} 35$ and may be suitable for decision analysis. ${ }^{36}$ To improve the actual application effect, the number needed to treat (NNT) will be estimated. The NNT will be evaluated using a control group risk of 30\%. Cohen's U3 index will be calculated to estimate the percentile gain in the intervention group. ${ }^{37}$
Data synthesis

Data synthesis will include the abstraction and pooling of results. Each study included from each meta-analysis will translate into an overall finding of similar results, such as overall sleep quality. RevMan V.5.3.5 will be used for data synthesis and analysis. The random effect and fixed effect models will be used to complete the summary of the research. If there is no significant heterogeneity $\left(\mathrm{I}^{2}<50 \%\right)$, a fixed effect model will be used; otherwise, if $\mathrm{I}^{2} \geq 50 \%$, a random effect model will be used. If the data cannot be merged due to essential heterogeneity, a descriptive analysis will be performed.

\section{Subgroup analysis}

If sufficient RCTs are included, we will conduct a subgroup analysis when there is significant heterogeneity in the study. We will conduct subgroup analyses of age, gender, sports fields (indoor vs outdoor), sample size, research time (4 weeks, 8 weeks, 12 weeks, 16 weeks and over 16 weeks), the existence of complications (hypertension, diabetes and cancer), the type of intervention, the type of sleep disturbance and the type of drug used.

\section{Sensitivity analysis}

Sensitivity analysis is performed to investigate the effect of one study. We will conduct a sensitivity analysis based on sample size, method quality, statistical model and lost data to evaluate the robustness of the main result indicators. After excluding low-quality research, the data will be merged and meta-analysed again.

\section{Patient and public involvement}

No patients or members of the public will be involved.

\section{Ethics and dissemination}

We do not use data related to personal data, so this protocol does not require ethical approval. The findings will be disseminated in peer-reviewed publications and journals.

\section{Limitations}

We acknowledge this protocol may suffer from limitations. We only search for studies written in Chinese and English and it is possible that the methodological quality of the eligible trials may be poor. Second, due to differences in exercise plans, there may be heterogeneity.

\section{DISCUSSION}

Sleep disturbance is a common problem in older adults ${ }^{38}$ and has significant negative effects on physical and mental health. Despite this, relatively few people seek treatment or consult a healthcare provider. ${ }^{4}$ Although there are regional differences in the types of treatments used to manage sleep disturbance, pharmacological agents still are the most widely used therapeutic option. However, at present, the first-line drugs used in clinical practice, such as diazepines, benzodiazepine receptor agonists, phytotherapeutic substances and melatonin, ${ }^{39}$ 
may cause side-effects in patients with long-term use. Therefore, exercise may be a useful alternative to improving sleep.

Previous reviews and meta-analyses have attempted to investigate the effects of exercise on insomnia. However, since most studies have only examined one or several small-scale trials, they have not been able to find out which exercise patterns are most effective for the elderly with sleep disturbances. This review and meta-analysis will provide a comprehensive review of RCTs to examine the effects of various exercise training programmes on sleep quality in the elderly with sleep disturbances. The study will determine the exercise patterns that have the least adverse reactions and are the most effective for older adults as well as providing objective evidence for the diagnosis and treatment of sleep disturbances.

The study may also reveal additional effects of exercise training in the elderly. For example, the root causes of sleep disturbances (such as depression and pain), the types of sleep disturbances (such as insomnia disorders, sleep-related breathing disorders, central disorders of hypersomnolence, circadian rhythm sleep-wake disorders) and the presence of underlying diseases (such as hypertension and diabetes) may influence the effects of exercise in the elderly. It is hoped that this meta-analysis will provide objective evidence for customised exercise prescriptions for the elderly with sleep disorders.

Acknowledgements We would like to thank our subject librarians for their help with the search strategy.

Contributors WZ, DL and WW conceived and designed the research; YL, YJ and DL wrote the first draft; JY, QZ, YG, ZW and WW reviewed and contributed to drafting, revising and finalising the manuscript. All authors have reviewed and approved the final version of the manuscript and have given their permission for publication.

Funding This work was supported financially by grants from Sichuan Science and Technology Program (nos. 20SYSX0293, 2020YFS0154, 2020YFSY0014, 2019JDRC0105), National Clinical Research Centre for Geriatrics (no. Z20191009), Sichuan University West China Hospital (nos. 2018HXFH001, 2018HXFH027, 20HXFH050) and West China School of Nursing, Sichuan University (no. HXHL19023).

Competing interests None declared.

Patient consent for publication Not applicable.

Provenance and peer review Not commissioned; externally peer reviewed.

Supplemental material This content has been supplied by the author(s). It has not been vetted by BMJ Publishing Group Limited (BMJ) and may not have been peer-reviewed. Any opinions or recommendations discussed are solely those of the author(s) and are not endorsed by BMJ. BMJ disclaims all liability and responsibility arising from any reliance placed on the content. Where the content includes any translated material, BMJ does not warrant the accuracy and reliability of the translations (including but not limited to local regulations, clinical guidelines, terminology, drug names and drug dosages), and is not responsible for any error and/or omissions arising from translation and adaptation or otherwise.

Open access This is an open access article distributed in accordance with the Creative Commons Attribution Non Commercial (CC BY-NC 4.0) license, which permits others to distribute, remix, adapt, build upon this work non-commercially, and license their derivative works on different terms, provided the original work is properly cited, appropriate credit is given, any changes made indicated, and the use is non-commercial. See: http://creativecommons.org/licenses/by-nc/4.0/.

ORCID iD

Wei Zhang http://orcid.org/0000-0002-8326-8496

\section{REFERENCES}

1 K Pavlova M, Latreille V. Sleep disorders. Am J Med 2019;132:292-9.

2 Sateia MJ. International classification of sleep disorders-third edition: highlights and modifications. Chest 2014;146:1387-94.

3 Ford ES, Cunningham TJ, Croft JB. Trends in self-reported sleep duration among US adults from 1985 to 2012. Sleep 2015;38:829-32.

4 Morin CM, LeBlanc M, Bélanger L, et al. Prevalence of insomnia and its treatment in Canada. Can J Psychiatry 2011;56:540-8.

5 Wolkove N, Elkholy O, Baltzan M, et al. Sleep and aging: 1. sleep disorders commonly found in older people. CMAJ 2007;176:1299-304

6 Kocevska D, Lysen TS, Dotinga A, et al. Sleep characteristics across the lifespan in 1.1 million people from the Netherlands, United Kingdom and United States: a systematic review and meta-analysis. Nat Hum Behav 2021;5:113-22.

7 Meng L, Zheng Y, Hui R. The relationship of sleep duration and insomnia to risk of hypertension incidence: a meta-analysis of prospective cohort studies. Hypertens Res 2013;36:985-95.

8 Antunes BM, Campos EZ, Parmezzani SS, et al. Sleep quality and duration are associated with performance in maximal incremental test. Physiol Behav 2017;177:252-6.

9 González-Rodríguez A, Labad J, Seeman MV. Sleep disturbances in patients with persistent delusions: prevalence, clinical associations, and therapeutic strategies. Clocks Sleep 2020;2:399-415

10 Cruz T, García L, Álvarez MA, et al. Sleep quality and memory function in healthy ageing. Neurologia 2019. doi:10.1016/j. nrl.2018.10.001. [Epub ahead of print: 11 Apr 2019].

11 Hein M, Lanquart J-P, Loas G, et al. Objective sleep alterations and long-term use of short or intermediate half-life benzodiazepine receptor agonists are risk factors for high blood pressure in individuals with insomnia: a study in 1272 individuals referred for sleep examinations. Sleep Med 2019;53:115-23.

12 Bathgate CJ, Fernandez-Mendoza J, Insomnia F-MJ. Insomnia, short sleep duration, and high blood pressure: recent evidence and future directions for the prevention and management of hypertension. Curr Hypertens Rep 2018;20:52.

13 Kowall B, Lehnich A-T, Strucksberg K-H, et al. Associations among sleep disturbances, nocturnal sleep duration, daytime napping, and incident prediabetes and type 2 diabetes: the Heinz Nixdorf recall study. Sleep Med 2016;21:35-41.

14 Anderson KO, Getto CJ, Mendoza TR, et al. Fatigue and sleep disturbance in patients with cancer, patients with clinical depression, and community-dwelling adults. J Pain Symptom Manage 2003;25:307-18.

15 Vgontzas AN, Liao D, Pejovic S, et al. Insomnia with short sleep duration and mortality: the Penn state cohort. Sleep 2010;33:1159-64.

16 Jiang B, He D, Guo Z, et al. Insomnia disorder increases the risk of mortality: pooled analysis of annual cumulative Time-to-Event data. Psychiatr Q 2020;91:1431-7.

17 Qaseem AKD, Forciea MA, et al. Clinical guidelines Committee of the American College of P. management of chronic insomnia disorder in adults: a clinical practice guideline from the American College of physicians. Ann Intern Med 2016;165:25-33.

18 Riemann D, Baglioni C, Bassetti C, et al. European guideline for the diagnosis and treatment of insomnia. J Sleep Res 2017;26:675-700.

19 Allain H, Bentué-Ferrer D, Polard E, et al. Postural instability and consequent falls and hip fractures associated with use of hypnotics in the elderly: a comparative review. Drugs Aging 2005;22:749-65.

20 Treves N, Perlman A, Kolenberg Geron L, et al. Z-drugs and risk for falls and fractures in older adults-a systematic review and metaanalysis. Age Ageing 2018;47:201-8.

21 Glass J, Lanctôt KL, Herrmann N, et al. Sedative hypnotics in older people with insomnia: meta-analysis of risks and benefits. BMJ 2005;331:1169.

22 Poluektov MG, Pchelina PV. [Insomnia due to hypnotic abuse]. Zh Nevrol Psikhiatr Im S S Korsakova 2016;116:24-9.

23 Mora JC, Valencia WM. Exercise and older adults. Clin Geriatr Med 2018;34:145-62.

24 Tai F, Wang C, Deng X, et al. Treadmill exercise ameliorates chronic REM sleep deprivation-induced anxiety-like behavior and cognitive impairment in C57BL/6J mice. Brain Res Bull 2020;164:198-207.

25 Yang $\mathrm{P}-\mathrm{Y}, \mathrm{Ho} \mathrm{K}-\mathrm{H}$, Chen $\mathrm{H}-\mathrm{C}$, et al. Exercise training improves sleep quality in middle-aged and older adults with sleep problems: a systematic review. J Physiother 2012;58:157-63.

26 Kelley GA, Kelley KS. Exercise and sleep: a systematic review of previous meta-analyses. J Evid Based Med 2017;10:26-36.

27 Montgomery P, Dennis J. Physical exercise for sleep problems in adults aged 60+. Cochrane Database Syst Rev 2002;2002:Cd003404. 
28 Du S, Dong J, Zhang H, et al. Taichi exercise for self-rated sleep quality in older people: a systematic review and meta-analysis. Int $J$ Nurs Stud 2015;52:368-79.

29 Vanderlinden J, Boen F, van Uffelen JGZ. Effects of physical activity programs on sleep outcomes in older adults: a systematic review. Int $J$ Behav Nutr Phys Act 2020;17:11.

30 Buysse DJ, Reynolds CF, Monk TH, et al. The Pittsburgh sleep quality index: a new instrument for psychiatric practice and research. Psychiatry Res 1989;28:193-213.

31 JM L. Overview of the self-rating sleep status scale (SRSS). Chinese $J$ Health Psychol 2012;20:1851.

32 Soldatos CR, Dikeos DG, Paparrigopoulos TJ. Athens insomnia scale: validation of an instrument based on ICD-10 criteria. $J$ Psychosom Res 2000;48:555-60.

33 Morin CM, Belleville G, Bélanger L, et al. The insomnia severity index: psychometric indicators to detect insomnia cases and evaluate treatment response. Sleep 2011;34:601-8.
34 Kelley GA, Kelley KS. Impact of progressive resistance training on lipids and lipoproteins in adults: another look at a meta-analysis using prediction intervals. Prev Med 2009;49:473-5.

35 Graham PL, Moran JL. Robust meta-analytic conclusions mandate the provision of prediction intervals in meta-analysis summaries. $J$ Clin Epidemiol 2012;65:503-10.

36 Ades AE, Caldwell DM, Reken S, et al. Evidence synthesis for decision making 7: a reviewer's checklist. Med Decis Making 2013;33:679-91.

37 Egger M, Davey Smith G, Schneider M, et al. Bias in meta-analysis detected by a simple, graphical test. BMJ 1997;315:629-34.

38 McHugh JE, Fan CW, Kenny RA, et al. Orthostatic hypotension and subjective sleep quality in older people. Aging Ment Health 2012;16:958-63.

39 Sateia MJ, Buysse DJ, Krystal AD, et al. Clinical practice guideline for the pharmacologic treatment of chronic insomnia in adults: an American Academy of sleep medicine clinical practice guideline. $J$ Clin Sleep Med 2017;13:307-49. 